PROCEEDINGS OF THE

AMERICAN MATHEMATICAL SOCIETY

Volume 133, Number 1, Pages 133-143

S 0002-9939(04)07603-8

Article electronically published on July 22, 2004

\title{
CIRCULAR AVERAGES AND FALCONER/ERDÖS DISTANCE CONJECTURE IN THE PLANE FOR RANDOM METRICS
}

\author{
S. HOFMANN AND A. IOSEVICH \\ (Communicated by Andreas Seeger)
}

\begin{abstract}
We study a variant of the Falconer distance problem for perturbations of the Euclidean and related metrics. We prove that Mattila's criterion, expressed in terms of circular averages, which would imply the Falconer conjecture, holds on average. We also use a technique involving diophantine approximation to prove that the well-distributed case of the Erdös Distance Conjecture holds for almost every appropriate perturbation of the Euclidean metric.
\end{abstract}

\section{INTRODUCTION}

Let $E \subset[0,1]^{d}, d \geq 2$. The celebrated Falconer conjecture says that if the Hausdorff dimension of $E$ exceeds $\frac{d}{2}$, then the distance set $\Delta(E)=\{|x-y|: x, y \in$ $E\}$ has positive Lebesgue measure.

The initial result in this direction was proved by Falconer ([Falconer86]) who showed that $\Delta(E)$ has positive Lebesgue measure if the Hausdorff dimension of $E$ exceeds $\frac{d+1}{2}$. This result was later improved in all dimensions by Bourgain ([Bourgain94]). The best known result in the plane is due to Tom Wolff, who proved that $\Delta(E)$ has positive Lebesgue measure provided that the Hausdorff dimension of $E$ is greater than $\frac{4}{3}$.

The main tool in Wolff's argument is the following estimate of the quantity introduced by Mattila ([Mattila87]):

$$
\int_{1}^{\infty}\left(\int_{S^{1}}|\widehat{\mu}(t \omega)|^{2} d \omega\right)^{2} t d t<\infty
$$

where $\mu$ is a Borel measure on a compact set $E$.

Wolff (Wolff99) obtained a best possible estimate for $\int_{S^{1}}|\widehat{\mu}(t \omega)|^{2} d \omega$ and used it to show that (0.1) holds if the Hausdorff dimension of $E$ is greater than $\frac{4}{3}$. An example due to Sjölin ([Sjolin93|) shows that Bourgain's estimate is best possible, so one cannot go below $\frac{4}{3}$ by restricting one's attention to $\int_{S^{1}}|\widehat{\mu}(t \omega)|^{2} d \omega$. However, it is still possible that one can establish (0.1) in the case when the Hausdorff dimension of $E$ is greater than 1 by exploiting the averaging in the $t$ variable in a non-trivial way. In this paper we shall prove that (0.1) and its variants hold on average (see the proof of Theorem 0.1 below), with respect to a system of dilations and rotations.

Received by the editors July 19, 2003 and, in revised form, September 8, 2003.

2000 Mathematics Subject Classification. Primary 42B10, 52C10.

The research of the authors was partially supported by NSF grants. 
Our analysis relies on averaging in the $t$ variable and cannot be carried out simply by obtaining average estimates for the square function $\int_{S^{1}}|\widehat{\mu}(t \omega)|^{2} d \omega$.

The Falconer distance conjecture is a continuous variant of the Erdös distance conjecture, which says that if $S$ is a finite set in the plane, then $\# \Delta(S) \geq C(\# S)^{1-\epsilon}$ for any $\epsilon>0$. The best known result in this direction is due to Katz and Tardos ( KatzTardos03]) who proved that $\# \Delta(S) \geq C(\# S) \approx .86$.

While an explicit link, on the level of exponents, between the Erdös distance problem and the Falconer distance problem is not available, in this paper we prove an explicit connection (see Corollary 0.2 below) between the Falconer distance conjecture and the following asymptotic version of the Erdös distance problem. Let $A$ be an infinite subset of the plane, separated in the sense that $\left|a-a^{\prime}\right| \geq c>0$ for any $a \neq a^{\prime} \in A$, and well-distributed in the sense that every square of sidelength $C>0$ contains at least one point of $A$. Then we ask for the estimate of the form $\Delta\left(A \cap[-R, R]^{2}\right) \geq C R^{\beta}, \beta>0$, as $R \rightarrow \infty$. If the Erdös distance conjecture is true, then $\beta=2-\epsilon$ for every $\epsilon>0$. A more precise description of this problem is given below. We shall see that any non-trivial result for the Falconer distance problem results in a corresponding estimate for this version of the Erdös distance problem to which we shall refer as the Asymptotic Erdös distance problem.

In this paper we shall also prove that the conclusion of the Falconer distance conjecture and the Asymptotic Erdös Distance Conjecture holds for almost every linear perturbation of the Euclidean and similar metrics. More precisely, let

$$
\Delta_{K}(E)=\left\{\|x-y\|_{K}: x, y \in E\right\},
$$

where $K$ is a symmetric bounded convex set in $\mathbb{R}^{2}$ and $\|\cdot\|_{K}$ is the distance induced by $K$. Our main results are the following.

Theorem 0.1. Let $E \subset[0,1]^{2}$ be a set of Hausdorff dimension greater than 1 . Let $K_{a, \phi}$ denote the ellipse centered at the origin with eccentricities $a_{1}, a_{2}, 1 \leq a_{j} \leq 2$, rotated by the angle $\phi$, and let $\Delta_{a, \phi}(E)$ denote the corresponding distance set. Then

$$
\int_{0}^{\pi} \iint_{1}^{\infty}\left(\int_{S^{1}}\left|\widehat{\mu}\left(t \omega_{a, \phi}\right)\right|^{2} d \omega\right)^{2} t d t \psi(a) d a d \phi<\infty
$$

where $\psi$ is a smooth cutoff function identically equal to 1 in $[1,2]^{2}$ and vanishing outside $[1 / 2,4]^{2}$, and $\omega_{a,-\phi}=\rho_{\phi}^{-1} \delta_{a} \omega$, where $\rho_{\phi}$ denotes the rotation by the angle $\phi, \delta_{a}(x)=\left(a_{1} x_{1}, a_{2} x_{2}\right)$, with $\omega$ on the unit circle.

It follows (see Theorem 1.1 and Theorem 1.2 below) that for almost every $(a, \phi) \in$ $[1,2]^{2} \times[0, \pi)$, the Lebesgue measure of $\Delta_{a, \phi}(E)$ is positive.

The connection between (0.3) and the second part of Theorem 0.1 is established by using Theorem 1.1 and Theorem 1.2 below.

Theorem 0.1 can be easily generalized to distances coming from convex symmetric planar sets $K$ with smooth well-curved boundaries. This amounts to modifying the estimate (2.10) using the fact that a smooth convex curve with non-vanishing curvature has second-order contact with its tangent lines. Detailed estimates of this and related types will appear in Arutyunyants04. We choose not to focus on this issue, since the main point of Theorem 0.1 is to prove an average version of the estimate (0.1) for the Euclidean metric for the purpose of shedding some light on the geometry of this inequality. 
The sharpness of the second part of Theorem 0.1 is demonstrated by a modification of a construction due to Falconer ([Falconer86]). Let $0<s \leq 2$. Let $q_{1}, q_{2}, \ldots, q_{i} \ldots$ be a sequence of positive integers such that $q_{i+1} \geq q_{i}^{i}$. Let $E_{i}=$ $\left\{x \in \mathbb{R}^{2}: 0 \leq x_{j} \leq 1,\left|x_{j}-p_{j} / q_{i}\right| \leq q_{i}^{-\frac{2}{s}}\right.$ for some integers $\left.p_{j}, j=1,2\right\}$. It is not hard to see (see e.g. WWolff02]) that the Hausdorff dimension of $E=\bigcap_{i=1}^{\infty} E_{i}$ is $s$. Also, $\Delta(E) \subset \bigcap_{i=1}^{\infty} \Delta\left(E_{i}\right)$.

Let $P_{i}=\left\{p=\left(p_{1}, p_{2}\right): 0 \leq p_{j} \leq q_{i}\right\}$. Let $\Delta_{a, \phi}^{(0,0)}\left(P_{i}\right)=\left\{\|p\|_{a, \phi}: p \in P_{i}\right\}$. It is immediate that $\# \Delta_{a, \phi}^{(0,0)}\left(P_{i}\right) \leq\left(q_{i}+1\right)^{2}$. By translation invariance it follows that $\# \Delta_{a, \phi}\left(P_{i}\right) \leq(q+1)^{2}$. We conclude that $\Delta_{a, \phi}\left(E_{i}\right)$ is contained in at most $\left(q_{i}+1\right)^{2}$ intervals of length $\approx q_{i}^{-\frac{2}{s}}$. It follows that the Hausdorff dimension of $\Delta(E)$ is $\leq s$. Thus if $s<1, \Delta_{a, \phi}(E)$ has Lebesgue measure 0 for every $a, \phi$.

Observe that in the above example it is not necessary for the $p_{i}$ 's to be integers. It is quite sufficient for $P_{i}$ to be sufficiently dense, and separated in the sense that there exists $c>0$ such that $\left|p-p^{\prime}\right| \geq c, p, p^{\prime} \in P_{i}, p \neq p^{\prime}$. This observation allows us to use Theorem 0.1 to deduce the aforementioned version of the Erdös distance conjecture. See e.g. PaAg95 for a thorough description of the Erdös Distance Problem and related concepts.

Asymptotic version of the Erdös Distance Conjecture. Let $A$ be a separated subset of $\mathbb{R}^{2}$. Suppose that $A$ is also well-distributed in the sense that there exists $r_{0}>0$ such that every cube of side-length $r_{0}$ contains at least one point of $A$. Then for any $\epsilon>0$ there exists a positive constant $C_{\epsilon}^{\prime}>0$ such that

$$
\# \Delta\left(A \cap[-R, R]^{2}\right) \geq C_{\epsilon}^{\prime} R^{2-\epsilon} .
$$

Using the above counter-example used to establish sharpness of Theorem 0.1 we can prove the following random variant of the asymptotic version of the Erdös Distance Conjecture. See IoLa2003 for a systematic application of this mechanism to non-Euclidean distances in $\mathbb{R}^{d}$.

Corollary 0.2. Let $A$ be as in the statement of the asymptotic version of the Erdös Distance Conjecture. Then for any $\epsilon>0$, there exists $C_{\epsilon}^{\prime}>0$ such that

$$
\# \Delta_{a, \phi}\left(A \cap[-R, R]^{2}\right) \geq C_{\epsilon}^{\prime} R^{2-\epsilon}
$$

for almost every $(a, \phi) \in[1,2]^{2} \times[0, \pi]$.

We prove Corollary 0.2 using what we call a diophantine conversion mechanism. Let $E_{i}, E$, and $\left\{q_{i}\right\}$ be defined as in the counter-example above with $P_{i}=A \cap\left[0, q_{i}\right]^{2}$. Suppose that $\# \Delta_{a, \phi}\left(P_{i}\right) \leq C q_{i}^{2-\epsilon}$ for some $\epsilon>0$ for a sequence of $i$ 's going to infinity. Then $\Delta_{a, \phi}\left(E_{i}\right)$ can be covered by $\leq C q_{i}^{2-\epsilon}$ intervals of length $\approx q^{-\frac{2}{s}}$. It follows that the Hausdorff dimension of $\Delta_{a, \phi}(E)$ is $\leq s-\frac{s \epsilon}{2}$. Now let $s=1+\delta$, $\delta>0$. We conclude that the Hausdorff dimension of $\Delta_{a, \phi}(E)$ is $\leq 1+\delta-\frac{(1+\delta) \epsilon}{2}<1$ if $\delta$ is sufficiently small. This is a contradiction because Theorem 0.1 implies that $\Delta_{a, \phi}(E)$ has positive Lebesgue measure for almost every $(a, \phi)$.

Remark 1. It has been pointed out to us that the second part of Theorem 0.1 can be proved using the Marstrand projection theorem (see, for example, Wolff02]). The outline is as follows. Let $E^{2}=\left\{\left(x_{1}^{2}, x_{2}^{2}\right): x \in E\right\}$, and let $E_{r}^{2}=\left\{\left(r x_{1}^{2}, x_{2}^{2}\right): x \in E\right\}$. One can show that the Hausdorff dimension of $E^{2}$ is the same as the Hausdorff dimension of $E$. In particular, if the Hausdorff dimension of $E$ is greater than one, 
then so is the Hausdorff dimension of $E^{2}$. The projection of $E_{r}^{2}$ onto a line of slope $r^{-1}$ is $r x_{1}^{2}+x_{2}^{2}$. By the Marstrand projection theorem, almost every such projection has positive Lebesgue measure, from which one can conclude that the second part of Theorem 0.1 holds without the need to consider rotations.

In the case of dilations and rotations of convex sets with smooth boundaries and non-vanishing curvature, an alternate approach is to use a modification of generalized projection theorems proved in Solomyak98 and PeresSchlag00. However, we choose to give a direct argument, which also proves an average version of (0.1).

Remark 2. Another way of stating Theorem 0.1 is to say that if $E \subset[0,1]^{2}$ is a set of Hausdorff dimension greater than 1, then for almost every linear transformation $T$ of the form rotation followed by an anisotropic dilation, $\Delta(T E)$ has positive Lebesgue measure.

Remark 3. In principle, an appropriate variant of Theorem 0.1 should hold in the context of two-dimensional Riemannian manifolds. We shall address this issue in a subsequent paper.

Remark 4. It is worth noting that large classes of two-dimensional sets of Hausdorff dimension $\alpha>1$ for which the Falconer conjecture holds can be constructed using more complicated probabilistic schemes. For example, let $A$ be a compact subset of the real line of Hausdorff dimension $\frac{1}{2}<\frac{\alpha}{2}<1$, and $W(t)$ an almost surely continuous version of the real Wiener process. A theorem due to J.P. Kahane (see e.g. Kahane68]) says that $E=W(A)$ is almost surely a Salem set of dimension $\alpha>1$, which means that $E$ is equipped with a Borel measure $\mu$ such that $|\widehat{\mu}(\xi)| \leq$ $C(1+|\xi|)^{-\frac{\alpha}{2}}$. Using Theorem 1.1 below one easily deduces that $\Delta(E)$ has positive Lebesgue measure, so the Falconer conjecture holds for this class of fractal sets.

Remark 5. In particular, the proof of Corollary 0.2 shows that the Falconer Distance Conjecture implies the asymptotic Erdös Distance Conjecture. It would be nice to prove that the Falconer conjecture in fact implies the standard Erdös Distance Conjecture. This amounts to eliminating the well-distributivity assumption on $A$ in the statement of Corollary 0.2 and replacing $C_{\epsilon}^{\prime} R^{2-\epsilon}$ on the right-hand side of $(0.2)$ by $C_{\epsilon}^{\prime}\left(\# A \cap[-R, R]^{2}\right)^{1-\epsilon}$.

\section{Method of PRoOf of TheOREM 0.1}

We use a modification of the following result due to Mattila ([Mattila87]).

Theorem 1.1. Let $E \subset[0,1]^{2}$ with a Borel measure $\mu$. Suppose that

$$
\int_{1}^{\infty}\left(\int_{S^{1}}|\widehat{\mu}(t \omega)|^{2} d \omega\right)^{2} t d t<\infty .
$$

Then $\Delta(E)$ has positive Lebesgue measure. (Here and throughout $\Delta(E)=$ $\Delta_{K}(E)$ with $K$ a unit disk.)

In fact, the argument used to prove Theorem 0.1 combined with a standard stationary phase argument (see e.g. Lemma 1.3 below) yields the following slightly more general result. 
Theorem 1.2. Let $E \subset[0,1]^{2}$ with Borel measure $\mu$. Let $K$ be a bounded convex set such that $\partial K$ is smooth and has everywhere non-vanishing curvature. Suppose that

$$
\int_{1}^{\infty}\left(\int_{\partial K}\left|\widehat{\mu}\left(t \omega_{K}\right)\right|^{2} d \omega_{K}\right)^{2} t d t<\infty
$$

where $d \omega_{K}$ denotes the Lebesgue measure on $\partial K$, the boundary of $K$.

Let $K^{*}=\left\{\xi: \sup _{x \in K} x \cdot \xi \leq 1\right\}$, the convex set dual to $K$. Then $\Delta_{K^{*}}(E)$ has positive Lebesgue measure.

We shall give a proof of Theorem 1.2 at the end of this paper for the sake of completeness.

As we noted above, in view of Theorem 1.1 and Theorem 1.2, the second part of Theorem 0.1 follows from (0.3).

The result due to Wolff mentioned above was proved by showing that under the assumptions of Theorem 0.1,

$$
\int_{S^{1}}|\widehat{\mu}(t \omega)|^{2} d \omega \lesssim t^{-\frac{\alpha}{2}}
$$

and an example due to Sjolin ([Sjolin93] ) shows that this estimate cannot, in general, be improved. This means that the proof of Theorem 0.3 must heavily rely on averaging in $t, a$, and $\phi$.

Throughout the paper we shall make use of the following version of the method of stationary phase. See e.g. Sogge93, Theorem 1.2.1.

Lemma 1.3. Let $S$ be a convex smooth hypersurface in $\mathbb{R}^{d}$ with everywhere nonvanishing Gaussian curvature and $d \mu$ a $C_{0}^{\infty}$ measure on $S$. Then

$$
|\widehat{d \mu}(\xi)| \lesssim|\xi|^{-\frac{d-1}{2}} .
$$

Moreover, suppose that $\Gamma \in \mathbb{R}^{d} \backslash(0, \ldots, 0)$ is the cone consisting of all vectors $\xi$ normal to $S$ at some point $x$ in a fixed relatively compact neighborhood of support of $d \mu$. Then

$$
\left|\left(\frac{\partial}{\partial \xi}\right)^{\alpha} \widehat{d \mu}(\xi)\right|=O\left((1+|\xi|)^{-N}\right) \forall N, \text { if } \xi \notin \Gamma,
$$

and

$$
\widehat{d \mu}(\xi)=\sum_{j=1}^{2} e^{-2 \pi i x_{j} \cdot \xi} a_{j}(\xi), \text { if } \xi \in \Gamma,
$$

where the finite sum is taken over the points $x_{j} \in \mathcal{N}$ having $\xi$ as a normal and

$$
\left|\left(\frac{\partial}{\partial \xi}\right)^{\alpha} a_{j}(\xi)\right| \leq C_{\alpha}(1+|\xi|)^{-\frac{d-1}{2}-|\alpha|} .
$$

Notation. Throughout this paper, $a \lesssim b$ means that there exists a positive constant $C$ such that $a \leq C b$. Similarly, $a \lesssim b$, with respect to a parameter $s$, means that given $\epsilon>0$ there exists $C_{\epsilon}>0$ such that $a \leq C_{\epsilon} s^{\epsilon} b$. 


\section{Proof of Theorem 1.3}

Let $\beta \in C_{0}^{\infty}$ be supported in $[1 / 2,4], \beta \equiv 1$ in $[1,2]$, and $\sum_{j} \beta\left(2^{-n} \cdot\right) \equiv 1$. Let $\omega_{a,-\phi}=\rho_{\phi}^{-1} \delta_{a} \omega$, where $\rho_{\phi}$ denotes the rotation by the angle $\phi$, and $\delta_{a}(x)=$ $\left(a_{1} x_{1}, a_{2} x_{2}\right)$. Let $\mu$ be a probability measure on $E$ such that $\mu(\{x \in E:|x-y| \leq$ $r\}) \leq C r^{\alpha}, r>0$, where $\alpha$ is the Hausdorff dimension of $E$. For the existence of such a measure, see, for example, Proposition 8.2 in Wolff02. Define

$$
\begin{gathered}
I_{n}=\iint\left(\int_{S^{1}}\left|\widehat{\mu}\left(t \omega_{a,-\phi}\right)\right|^{2} d \omega\right)^{2} t \beta\left(2^{-n} t\right) d t \psi(a) d a d \phi \\
=\iiint \int\left|\widehat{\mu}\left(t \omega_{a,-\phi}\right)\right|^{2}\left|\widehat{\mu}\left(t \omega_{a,-\phi}^{\prime}\right)\right|^{2} d \sigma(\omega) d \sigma\left(\omega^{\prime}\right) t \beta\left(2^{-n} t\right) d t \psi(a) d a d \phi
\end{gathered}
$$

$=\iiint \iint e^{2 \pi i\left((x-y) \cdot t \omega_{a,-\phi}^{\prime}+\left(x^{\prime}-y^{\prime}\right) \cdot t \omega_{a,-\phi}^{\prime}\right)} d \mu^{*} d \sigma(\omega) d \sigma\left(\omega^{\prime}\right) t \beta\left(2^{-n} t\right) d t \psi(a) d a d \phi$,

where

$$
d \mu^{*}=d \mu(x) d \mu(y) d \mu\left(x^{\prime}\right) d \mu\left(y^{\prime}\right),
$$

and $d \sigma$ denotes a $C_{0}^{\infty}$ measure on the sphere. Using a partition of unity we see that it is enough to consider this situation.

Integrating in $\omega$ and $\omega^{\prime}$ first, we get

$$
\iiint \int \widehat{\sigma}\left(t(x-y)_{a, \phi}\right) \widehat{\sigma}\left(t\left(x^{\prime}-y^{\prime}\right)_{a, \phi}\right) d \mu^{*} \beta\left(2^{-n} t\right) d t \psi(a) d a d \phi
$$

where $\sigma$ is a smooth cut-off function times the arc-length measure on $S^{1}$ as above, and $x_{a}=\left(a_{1}\left(x_{1} \cos (\phi)-x_{2} \sin (\phi)\right), a_{2}\left(x_{1} \sin (\phi)+x_{2} \cos (\phi)\right)\right)$.

Case 1: $2^{n}|x-y| \lesssim 1$ and $2^{n}\left|x^{\prime}-y^{\prime}\right| \lesssim 1$. Then for any $\epsilon>0,(1.3)$ is bounded by

$$
\int|x-y|^{-\alpha+\epsilon}\left|x^{\prime}-y^{\prime}\right|^{-\alpha+\epsilon} t^{-2 \alpha+2 \epsilon} d \mu(x) d \mu(y) d \mu\left(x^{\prime}\right) d \mu\left(y^{\prime}\right) t d t<\infty,
$$

as desired if $\epsilon$ is sufficiently small.

Case 2: $2^{n}|x-y|>>1$ and $2^{n}\left|x^{\prime}-y^{\prime}\right|>>1$. Observe that the symbol of order 0 resulting from pulling $(t|x-y|)^{-\frac{1}{2}}$ from the symbol $a_{j}$ given by Theorem 1.4 can be incorporated into the smooth cut-off $\beta$ without affecting the size or the support of $\beta$ or its derivatives. We shall suppress (harmless) dependence of $\beta$ on $x, y, x^{\prime}, y^{\prime}, a, \phi$ in what follows. Using this observation and (1.7) above, we see that (2.3) can be written as a sum of terms of the form

$$
\iiint \int e^{2 \pi i t\left(|x-y|_{a, \phi}-\left|x^{\prime}-y^{\prime}\right|_{a, \phi}\right)} t^{-1}|x-y|_{a, \phi}^{-\frac{1}{2}}\left|x^{\prime}-y^{\prime}\right|_{a, \phi}^{-\frac{1}{2}} t d \mu^{*} \beta\left(2^{-n} t\right) d t \psi(a) d a d \phi .
$$

We must also consider the term where $2 \pi i t\left(|x-y|_{a, \phi}+\left|x^{\prime}-y^{\prime}\right|_{a, \phi}\right)$ is the phase function, but this case is very easy. Let $\eta$ be a small parameter to be determined 
later. We have

$$
\begin{gathered}
\left|\iiint \int e^{2 \pi i t\left(|x-y|_{a, \phi}+\left|x^{\prime}-y^{\prime}\right|_{a, \phi}\right)} t^{-1}\right| x-\left.y\right|_{a, \phi} ^{-\frac{1}{2}}\left|x^{\prime}-y^{\prime}\right|_{a, \phi}^{-\frac{1}{2}} t d \mu^{*} \beta\left(2^{-n} t\right) d t \psi(a) d a d \phi \mid \\
\lesssim 2^{n} \int_{\left\{\left(x, y, x^{\prime}, y^{\prime}\right):|| x-y|+| x^{\prime}-y^{\prime}|| \lesssim 2-n(1-\eta)\right\}}|x-y|^{-\frac{1}{2}}\left|x^{\prime}-y^{\prime}\right|^{-\frac{1}{2}} d \mu^{*}+O\left(2^{n} 2^{-n \eta N}\right) \\
\quad \lesssim 2^{n} \int|x-y|^{-\alpha+\epsilon} 2^{-n(1-\eta)\left(\alpha-\frac{1}{2}-\epsilon\right)}\left|x^{\prime}-y^{\prime}\right|^{-\alpha+\epsilon} 2^{-n(1-\eta)\left(\alpha-\frac{1}{2}-\epsilon\right)} d \mu^{*} \\
\vdots 2^{2 n(1-\eta)} 2^{-2 n(1-\eta) \alpha} 2^{2 n(1-\eta) \epsilon},
\end{gathered}
$$

which sums if $\epsilon<\alpha-1$ and $\eta<1$. The second line of (2.6) follows from the first using the fact, which follows easily by integration by parts, that the Fourier transform of a smooth compactly supported function decays rapidly at infinity.

We now turn our attention to (2.5). Integrating in $t$ first we get

$$
2^{n} \iiint \widehat{\beta}\left(2^{n}\left(|x-y|_{a, \phi}-\left|x^{\prime}-y^{\prime}\right|_{a, \phi}\right)\right)|x-y|_{a, \phi}^{-\frac{1}{2}}\left|x^{\prime}-y^{\prime}\right|_{a, \phi}^{-\frac{1}{2}} \psi(a) d a d \phi d \mu^{*} .
$$

Let $I_{n, k, k^{\prime}}$ denote the quantity (2.7) localized to the sets where $2^{-k} \leq|x-y|_{a, \phi} \leq$ $2^{-k+1}, 2^{-k^{\prime}} \leq\left|x^{\prime}-y^{\prime}\right|_{a, \phi} \leq 2^{-k^{\prime}+1}$. We obtain

$$
I_{n, k, k^{\prime}} \approx 2^{n} 2^{\frac{k}{2}} 2^{\frac{k^{\prime}}{2}} \iiint\left|\widehat{\beta}\left(2^{n}\left(|x-y|_{a, \phi}-\left|x^{\prime}-y^{\prime}\right|_{a, \phi}\right)\right)\right| \psi(a) d a d \phi d \mu^{*} .
$$

Let $x-y=|x-y| e^{i A}, x^{\prime}-y^{\prime}=\left|x^{\prime}-y^{\prime}\right| e^{i B}$. We now decompose $x-y$ and $x^{\prime}-y^{\prime}$ into sectors of aperture $\delta$ to be determined. Let $S_{\delta}^{j, k}$ denote the "rectangle" formed by intersection of the annulus $\left\{z: 2^{-k} \leq|z| \leq 2^{-k+1}\right\}$ and the angular sector $\{z: j \delta \leq A \leq(j+1) \delta\}$. Define $S_{\delta}^{j^{\prime}}, k^{\prime}$ analogously.

Let

$$
\begin{gathered}
I_{n, k, k^{\prime}}^{j, j^{\prime}, \delta}=2^{n} 2^{\frac{k}{2}} 2^{\frac{k^{\prime}}{2}} \int_{S_{\delta}^{j, k} \otimes S_{\delta}^{j^{\prime}, k^{\prime}}} \iint\left|\widehat{\beta}\left(2^{n}\left(|x-y|_{a, \phi}-\left|x^{\prime}-y^{\prime}\right|_{a, \phi}\right)\right)\right| \psi(a) d a d \phi d \mu^{*} \\
=2^{n+\frac{k}{2}+\frac{k^{\prime}}{2}} \int_{\left\{x-y \in S_{\delta}^{j, k} ; x^{\prime}-y^{\prime} \in S_{\delta}^{j^{\prime}, k^{\prime}} ;|| x-\left.y\right|_{a, \phi}-\left|x^{\prime}-y^{\prime}\right|_{a, \phi} \mid \lesssim 2^{-n(1-\eta)\}}\right.} \iint \psi(a) d a d \phi d \mu^{*} \\
+O\left(2^{n} 2^{\frac{k}{2}} 2^{\frac{k^{\prime}}{2}} 2^{-n \eta N}\right),
\end{gathered}
$$

where $\eta>0$ is a small parameter to be chosen later.

Observe that for $j, j^{\prime}$ fixed, we have that $x-y$ is in a $\delta 2^{-k}$ by $2^{-n(1-\eta)}$ rectangle and $x^{\prime}-y^{\prime}$ is in a $\delta 2^{-k^{\prime}}$ by $2^{-n(1-\eta)}$ rectangle. Also observe that if both $k, k^{\prime} \gtrsim$ $n(1-\eta)$, then we have a simple estimate analogous to the one in Case 1 if $\eta$ is chosen to be sufficiently small and $\delta \approx 1$. Otherwise, if at least one of $k, k^{\prime}<<n(1-\eta)$, then they both are, and, moreover, $k \approx k^{\prime}$. Therefore, in what follows we may assume that we are in the latter situation, so that the double index appearing above may now be replaced by the single index $k$.

Now, multiplying both sides by ||$x-\left.y\right|_{a, \phi}+\left|x^{\prime}-y^{\prime}\right|_{a, \phi} \mid$ and computing the area of the resulting set, we see that

$$
\left|\left\{\left(a_{1}, a_{2}\right):|| x-\left.y\right|_{a, \phi}-\left|x^{\prime}-y^{\prime}\right|_{a, \phi} \mid \lesssim 2^{-n(1-\eta)}\right\}\right| \lesssim 2^{-(n(1-\eta)-k)}[|A-B||A+\phi|]^{-1}
$$


where, without loss of generality, $A \geq B$, so that $j-j^{\prime} \geq 1$ unless $j=j^{\prime}$. We also take $A, B, \phi$ to be small and positive. (The other cases follow by the same argument.) It follows that if $j \neq j^{\prime}$,

$$
I_{n, k}^{j, j^{\prime}, \delta} \lesssim 2^{n+k} \int_{S} \int \max \left\{1, \frac{2^{-(n(1-\eta)-k)}}{\left|j-j^{\prime}\right| \delta|\delta j+\phi|}\right\} d \phi d \mu^{*}
$$

where integration is over the set $S$ where $x-y \in S_{\delta}^{j, k}, x^{\prime}-y^{\prime} \in S_{\delta}^{j^{\prime}, k^{\prime}}$ (recall that $k \approx k^{\prime}$ ). In what follows the dependence of $S$ on parameters will be suppressed for notational convenience. Let $\delta \approx 2^{-n(1-\eta)+k}$. Then for $j^{\prime}$ and $x-y$ fixed, we have that $x^{\prime}-y^{\prime}$ is located in a ball of radius $\approx 2^{-n(1-\eta)}$. We have

$$
\begin{aligned}
I_{n, k}^{j, j^{\prime}} & \lesssim 2^{n+k} \int_{S} \int \max \left\{1, \frac{1}{\left|j-j^{\prime}\right||\delta j+\phi|}\right\} d \phi d \mu^{*} \\
& \lesssim 2^{n+k} \frac{1}{\left|j-j^{\prime}\right|} \int_{S} d \mu(x) d \mu(y) d \mu\left(x^{\prime}\right) d \mu\left(y^{\prime}\right) .
\end{aligned}
$$

We must estimate

$$
\sum_{k, n} 2^{n+k} \sum_{j, j^{\prime}} \frac{1}{\left|j-j^{\prime}\right|} \int_{S} d \mu(x) d \mu(y) d \mu\left(x^{\prime}\right) d \mu\left(y^{\prime}\right)
$$

Let $l=j-j^{\prime}$. We get

$$
\sum_{k, n} 2^{n+k} \sum_{l} \frac{1}{l} \sum_{j^{\prime}} \int_{S} d \mu(x) d \mu(y) d \mu\left(x^{\prime}\right) d \mu\left(y^{\prime}\right) .
$$

For a fixed $x-y$ and a given sector indexed by $j, x^{\prime}-y^{\prime}$ is contained in a ball of radius $C 2^{-n(1-\eta)}$ since $\delta 2^{-k} \approx 2^{-n(1-\eta)}$. Fixing $y^{\prime}$ and integrating in $x^{\prime}$ we get $2^{-n(1-\eta) \alpha}$ since $\mu$ is $\alpha$-dimensional. Taking the union over all the sectors indexed by $j^{\prime}$, we have $x-y$ in the annulus of width $2^{-k}$. Fixing $y$ and integrating in $x$, we pick up $C 2^{-k \alpha}$. It follows that (2.14) is bounded by a constant multiple of

$$
\sum_{k, n} 2^{n+k} \sum_{l} \frac{1}{l} 2^{-n(1-\eta) \alpha} 2^{-k \alpha} \lesssim 1
$$

if $(1-\eta) \alpha>1$, since $l$ runs up to $C 2^{n(1-\eta)-k}$, the number of sectors.

If $j=j^{\prime},(2.14)$ takes the form

$$
\sum_{k, n} 2^{n+k} \sum_{j^{\prime}} \int_{S} d \mu(x) d \mu(y) d \mu\left(x^{\prime}\right) d \mu\left(y^{\prime}\right),
$$

which is bounded by the same argument. 
Case 3: $2^{n}|x-y|>>1$ and $2^{n}\left|x^{\prime}-y^{\prime}\right| \lesssim 1$. This case is basically vacuous, which can be seen as follows. We have

$$
\begin{gathered}
\iiint \int e^{2 \pi i t\left(|x-y|_{a, \phi}-\left|x^{\prime}-y^{\prime}\right|_{a, \phi}\right)} t^{-\frac{1}{2}}|x-y|_{a, \phi}^{-\frac{1}{2}} t d \mu^{*} \beta\left(2^{-n} t\right) d t \psi(a) d a d \phi \\
=2^{n} 2^{\frac{n}{2}} \int_{\left\{2^{n}\left|x^{\prime}-y^{\prime}\right| \lesssim 1\right\}} \iint \widehat{\beta}\left(2^{n}\left(|x-y|_{a, \phi}-\left|x^{\prime}-y^{\prime}\right|_{a, \phi}\right)\right)|x-y|_{a, \phi}^{-\frac{1}{2}} \psi(a) d a d \phi d \mu^{*} \\
\lesssim 2^{n} 2^{\frac{n}{2}} \int_{\left\{2^{n}\left|x^{\prime}-y^{\prime}\right| \lesssim 1\right\}} \iint \widehat{\beta}\left(2^{n}\left(|x-y|_{a, \phi}\right)\right)|x-y|_{a, \phi}^{-\frac{1}{2}} \psi(a) d a d \phi d \mu^{*} \\
\quad \lesssim 2^{n} 2^{\frac{n}{2}} 2^{-n \alpha} \iint \widehat{\beta}\left(2^{n}(|x-y|)\right)|x-y|^{-\frac{1}{2}} d \mu(x) d \mu(y) .
\end{gathered}
$$

Localizing to the sets where $2^{-k} \leq|x-y| \leq 2^{-k+1}$, we obtain

$$
2^{n} 2^{\frac{n}{2}} 2^{-n \alpha} 2^{\frac{k}{2}} \iint \widehat{\beta}\left(2^{n}(|x-y|)\right) d \mu(x) d \mu(y) \lesssim 2^{n} 2^{\frac{n}{2}} 2^{-n \alpha} 2^{\frac{k}{2}} 2^{-n \alpha},
$$

which sums since $k<<n$. This completes the proof of Theorem 0.3, and, consequently, the proof of Theorem 0.1 .

\section{Proof of Theorem 1.2}

Define the measure $\nu_{0}$ by

$$
\int f d \nu_{0}=\int f\left(\|x-y\|_{K^{*}}\right) d \mu(x) d \mu(y)
$$

Let

$$
d \nu(s)=e^{i \frac{\pi}{4}} s^{-\frac{1}{2}} d \nu_{0}(s)+e^{-i \frac{\pi}{4}}|s|^{-\frac{1}{2}} d \nu_{0}(-s) .
$$

Since $\nu_{0}$ is supported on $\Delta_{K^{*}}(E), \nu$ is supported on $\Delta_{K^{*}}(E) \cup-\Delta_{K^{*}}(E)$.

We have

$$
\int_{\partial K}\left|\widehat{\mu}\left(t \omega_{K}\right)\right|^{2} d \omega_{K}=\int \widehat{\sigma}_{t} * \mu d \mu
$$

where $\sigma$ is the measure on $\partial K$.

Using a variant of Theorem 1.4 (see e.g. [Herz62]), we see that

$$
\widehat{\sigma}_{t}(x)=2\left(t \rho^{*}(x)\right)^{-\frac{1}{2}} \cos \left(2 \pi\left(t \rho^{*}(x)-\frac{1}{8}\right)\right)+O\left((t|x|)^{-\frac{3}{2}}\right),
$$

where

$$
\rho^{*}(x)=\sup _{y \in \partial K} x \cdot y .
$$

In other words, $\rho^{*}(x)=\|x\|_{K^{*}}$.

By definition,

$$
\begin{gathered}
\hat{\nu}(k)=e^{i \frac{\pi}{4}} \int\|x-y\|_{K^{*}}^{-\frac{1}{2}} e^{-2 \pi i k\|x-y\|_{K^{*}}} d \mu(x) d \mu(y) \\
+e^{-i \frac{\pi}{4}} \int\|x-y\|_{K^{*}}^{-\frac{1}{2}} e^{2 \pi i k\|x-y\|_{K^{*}}} d \mu(x) d \mu(y) \\
=2 \int\|x-y\|_{K^{*}}^{-\frac{1}{2}} \cos \left(2 \pi\left(|k| \rho^{*}(x-y)-\frac{1}{8}\right)\right) d \mu(x) d \mu(y) .
\end{gathered}
$$


By (3.3),

$$
\begin{gathered}
\int\left|\widehat{\mu}\left(k \omega_{K}\right)\right|^{2} d \omega_{K}=|k|^{-\frac{1}{2}} \int 2|| x-\left.y\right|_{K^{*}} ^{-\frac{1}{2}} \cos \left(2 \pi\left(|k| \rho^{*}(x-y)-\frac{1}{8}\right)\right) d \mu(x) d \mu(y) \\
+O\left(\int_{|x-y| \geq|k|^{-1}}(|k||x-y|)^{-\frac{3}{2}} d \mu(x) d \mu(y)\right) \\
+O\left(\int_{|x-y| \leq|k|^{-1}}(|k||x-y|)^{-\frac{1}{2}} d \mu(x) d \mu(y)\right) \\
=|k|^{-\frac{1}{2}} \int 2|| x-\left.y\right|_{K^{*}} ^{-\frac{1}{2}} \cos \left(2 \pi\left(|k| \rho^{*}(x-y)-\frac{1}{8}\right)\right) d \mu(x) d \mu(y) \\
+O\left(\int(|k||x-y|)^{-\frac{1}{2}} d \mu(x) d \mu(y)\right)
\end{gathered}
$$

for any $\alpha \in[1 / 2,3 / 2]$. It follows that

$$
\hat{\nu}(k)=|k|^{\frac{1}{2}} \int|\widehat{\mu}(k \omega)|^{2} d \omega+O\left(|k|^{\frac{1}{2}-\alpha} I_{\alpha}(\mu)\right) .
$$

Since the error term is clearly in $L^{2}(|k| \geq 1)$, we see that $\hat{\nu} \in L^{2}(|k| \geq 1)$ if and only if $|k|^{\frac{1}{2}} \int\left|\widehat{\mu}\left(k \omega_{K}\right)\right|^{2} d \omega_{K} \in L^{2}(|k| \geq 1)$. This is precisely what Theorem 1.2 asserts.

\section{REFERENCES}

[Arutyunyants04] G. Arutyunyants, Geometric methods in harmonic analysis, Doctoral Dissertation (in preparation) (2004).

[Bourgain94] J. Bourgain, Hausdorff dimension and distance sets, Israel J. Math. 87 (1994), 193-201. MR.1286826 (95h:28008)

[Falconer86] K. J. Falconer, On the Hausdorff dimensions of distance sets, Mathematika 32 (1986), 206-212. MR0834490 (87j:28008)

[Herz62] C. Herz, Fourier transforms related to convex sets, Ann. of Math. 75 (1962), 81-92. MR0142978 (26:545)

[IoLa2003] A. Iosevich and I. Łaba, K-distance sets and Falconer conjecture, (in preparation) (2002).

[Kahane68] J. P. Kahane, Some random series of functions, D. C. Heath and Co. Raytheon Education Co., Lexington, Mass. (1968). MR0254888 (40:8095)

[KatzTardos03] N. Katz and G. Tardos, On the Erdös Distance Problem, (preprint) (2003).

[Mattila87] P. Mattila, Spherical averages of Fourier transforms of measures with finite energy: dimensions of intersections and distance sets, Mathematika 34 (1987), 207-228. MR0933500 (90a:42009)

[PaAg95] J. Pach and and P. Agarwal, Combinatorial Geometry, Wiley-Interscience Series (1995). MR 1354145 (96j:52001)

[PeresSchlag00] Y. Peres and W. Schlag, Smoothness of projections, Bernoulli convolutions, and the dimension of exceptions, Duke Math. J. 102 (2000), 193-251. MR1749437 (2001d:42013)

[Sogge93] C. D. Sogge, Fourier integrals in classical analysis, Cambridge University Press (1993). MR 1205579 (94c:35178)

[Solomyak98] B. Solomyak, Measure and dimension for some fractal families, Math. Proc. Cambridge Philos. Soc. 124 (1998), 531-546. Mr.1636589 (99e:28016)

[Sjolin93] P. Sjölin, Estimates of spherical averages of Fourier transforms and dimensions of sets, Mathematika 40 (1993), 322-330. MR.1260895 (95f:28007) 
[Wolff99] T. Wolff, Decay of circular means of Fourier transforms of measures, Internat. Math. Res. Notices 10 (1999), 547-567. MR1692851 (2000k:42016)

[Wolff02] T. Wolff, Lectures on Harmonic Analysis, California Institute of Technology Class Lectures Notes (revised by I. Łaba) http://www.math.ubc.ca (2002). University Lecture Series, no. 29, Amer. Math. Soc. (2003). MR2003254 (2004e:42002)

Department of Mathematics, University of Missouri, Columbia, Missouri 65211

E-mail address: hofmann@math.missouri.edu

Department of Mathematics, University of Missouri, Columbia, Missouri 65211

E-mail address: iosevich@math.missouri.edu 